\title{
The Dual Effects of Reactive Oxygen Species on the Mandibular Alveolar Bone Formation in SOD1 Knockout Mice: Promotion or Inhibition
}

\author{
Yunyan Zhang $\mathbb{D}^{1,2,3,4}$ Yuzhi Yang $\mathbb{D}^{1,2,3,4}$ Mingxue $X u \mathbb{D}^{1,1,2,3,4}$ Jingwen Zheng $\mathbb{D}^{1,1,2,3,4}$ \\ Yuchan Xu $\mathbb{D}$, , $1,3,4$ Guoqing Chen $\mathbb{D},{ }^{1,2,3}$ Qiang Guo $\mathbb{D},^{3}$ Weidong Tian $\mathbb{D}^{1,2,3,5}$ \\ and Weihua Guo $\mathbb{D}^{1,2,3,4}$ \\ ${ }^{1}$ Engineering Research Center of Oral Translational Medicine, Ministry of Education, West China Hospital of Stomatology, \\ Sichuan University, Chengdu, China \\ ${ }^{2}$ National Engineering Laboratory for Oral Regenerative Medicine, West China Hospital of Stomatology, Sichuan University, \\ Chengdu, China \\ ${ }^{3}$ State Key Laboratory of Oral Diseases, National Clinical Research Center for Oral Diseases, West China Hospital of Stomatology, \\ Sichuan University, Chengdu, China \\ ${ }^{4}$ Department of Pediatric Dentistry, West China Hospital of Stomatology, Sichuan University, Chengdu, China \\ ${ }^{5}$ Department of Oral and Maxillofacial Surgery, West China Hospital of Stomatology, Sichuan University, Chengdu, China
}

Correspondence should be addressed to Weidong Tian; drtwd@sina.com and Weihua Guo; guoweihua943019@163.com

Received 29 August 2020; Revised 14 December 2020; Accepted 15 January 2021; Published 4 February 2021

Academic Editor: Rajeshwary Ghosh

Copyright (C) 2021 Yunyan Zhang et al. This is an open access article distributed under the Creative Commons Attribution License, which permits unrestricted use, distribution, and reproduction in any medium, provided the original work is properly cited.

The status of reactive oxygen species (ROS) correlates closely with the normal development of the oral and maxillofacial tissues. Oxidative stress caused by ROS accumulation not only affects the development of enamel and dentin but also causes pathological changes in periodontal tissues (periodontal ligament and alveolar bone) that surround the root of the tooth. Although previous studies have shown that ROS accumulation plays a pathologic role in some oral and maxillofacial tissues, the effects of ROS on alveolar bone development remain unclear. In this study, we focused on mandibular alveolar bone development of mice deficient in superoxide dismutase1 (SOD1). Analyses were performed using microcomputerized tomography (micro-CT), TRAP staining, immunohistochemical (IHC) staining, and enzyme-linked immunosorbent assay (ELISA). We found for the first time that slightly higher ROS in mandibular alveolar bone of SOD1(-/-) mice at early ages (2-4 months) caused a distinct enlargement in bone size and increased bone volume fraction (BV/TV), trabecular thickness (Tb.Th), and expression of alkaline phosphatase (ALP), Runtrelated transcription factor 2 (Runx2), and osteopontin (OPN). With ROS accumulation to oxidative stress level, increased trabecular bone separation (Tb.Sp) and decreased expression of ALP, Runx2, and OPN were found in SOD1(-/-) mice at 6 months. Additionally, dosing with N-acetylcysteine (NAC) effectively mitigated bone loss and normalized expression of ALP, Runx2, and OPN. These results indicate that redox imbalance caused by SOD1 deficiency has dual effects (promotion or inhibition) on mandibular alveolar bone development, which is closely related to the concentration of ROS and the stage of growth. We present a valuable model here for investigating the effects of ROS on mandibular alveolar bone formation and highlight important roles of ROS in regulating tissue development and pathological states, illustrating the complexity of the redox signal.

\section{Introduction}

Reactive oxygen species (ROS), a natural product of cellular aerobic metabolism, are chemically reactive molecules such as superoxide $\left(\mathrm{O}_{2}{ }^{-\cdots}\right)$, hydrogen peroxide $\left(\mathrm{H}_{2} \mathrm{O}_{2}\right)$, and the hydroxyl radical $\left(\mathrm{HO}^{\prime}\right)$. In earlier studies, oxidative stress caused by ROS accumulation has been linked to multisystem diseases associated with inflammation and aging. Recent 
studies have found that ROS play several important roles in cancer therapy, immunity, autophagy, and, especially, signal transduction [1-3]. In the low range, ROS perform complex signaling functions as a signaling molecule that regulates cell growth and differentiation [4]. However, high-concentration ROS may also contribute to oxidative denaturation of intracellular proteins and lipids, DNA damage, alteration of cell signaling pathways, and cell apoptosis and ultimately lead to the body's aging [5]. To regulate ROS concentrations, the body has an antioxidant enzyme system and antioxidant substances that eliminate ROS to achieve balance. It is well established that SODs are the first line of defense against ROS by catalyzing the disproportionation of superoxide radicals into hydrogen peroxide and molecular oxygen. There are three known SOD subtypes classified mainly by location in mammals: SOD1 present in cytoplasm and mitochondrial intermembrane, SOD2 localized at the mitochondrial matrix, and SOD3 being secreted to the extracellular space [6]. Most abundant and expressed in the majority of tissues, SOD1 has a surprisingly high cellular concentration [7] (estimated to be 10-40 $\mu \mathrm{M})$. Moreover, unlike SOD2(-/-) mice, homozygous mutations in the SOD1 gene are not lethal; still, SOD1(-/-) mice exhibit subtle defects, including Amyotrophic Lateral Sclerosis (ALS), retinal dysfunction, and muscle atrophy [8-11]. Therefore, SOD1-deficient mice are a verified animal model for studying the effects of ROS aggregation on tissue/organ development and disease occurrence and treatment.

Models established by methods such as a high-fat diet [12], stimulation of cells with sodium fluoride [13], and $\mathrm{H}_{2} \mathrm{O}_{2}$ dosage $[14,15]$ showed that oxidative stress can cause alterations in oral and maxillofacial tissues, causing premature senescence of dental pulp cells [15], apoptosis of enamel cells, interference of tooth mineralization [12-14], abnormal tooth development, periodontal diseases [15], and oral cancer [16]. Previous studies have demonstrated that SOD1 knockout mice showed bone disturbances. A study of the femur found that in SOD1-deficient subjects, ROS accumulation caused reduced bone formation, absorption, and low bone remodeling activity, which resulted in accelerated bone loss and increased bone fragility [17]. Another study demonstrated that femurs of SOD1 knockout mice were weaker than those of wild-type mice during puberty skeletal growth [18]. Furthermore, ROS have been implicated in bone metabolism by mediating osteoclastic resorption [19] and osteoblastic differentiation [20]. Excessive ROS may cause apoptosis of osteoblasts and consequently bone metabolic disorders [21].

The alveolar bone and teeth develop closely and together form the dentoalveolar complex. The alveolar bone anchors the teeth. Disruptions in alveolar bone development and maintenance can result in pathological changes such as bone loss or osteoporosis, which may lead to mobile teeth and maxillofacial bone diseases, affecting physiological function as well as aesthetics. Excessive ROS accumulation has an adverse impact on the development of long bones and some oral and maxillofacial tissues. However, research on the effects of ROS on the development of mandibular alveolar bone is absent. Hence, we hypothesized that ROS aggregation impacts mandibular alveolar bone formation. Specifically, we established a SOD1 knockout mouse model and demonstrated that ROS aggregation could have dual effects on mandible formation: promotion or pathological effect, which depended on ROS levels and development stage. Furthermore, treatment with the antioxidant NAC effectively improved bone loss and increased expression of osteogenesis protein markers.

\section{Materials and Methods}

All animal experiments were conducted in accordance with the principles and procedures of the National Institutes of Health (NIH) Guide for the Care and Use of Laboratory Animals and approved by the Ethics Committee of the State Key Laboratory of Oral Diseases, West China Hospital of Stomatology (Chengdu, Sichuan) (license number WCHSIRB-D2016-195).

2.1. Experimental Animals, Genotyping, and Grouping. SOD1(-/-) mice and corresponding SOD1(+/-) and SOD1 $(+/+)$ controls were generated from the intercross between congenic B6 <Sod1+/->; 129S-Sod1tm1Leb/J, purchased from Shanghai Southern Model Biotechnology Co., Ltd. All mice were housed in the pathogen-free facility at Sichuan University Experimental Animal Center and given SPF animal feed and drinking water. The photoperiod was carried out according to the law of 12:12 circadian rhythm. The illumination intensity was 130-325 lux, the room temperature was $21 \pm 3^{\circ} \mathrm{C}$, the relative humidity was $40-60 \%$, the noise was $25-40 \mathrm{~dB}$, and the air exchange times were 10-15 times/hour. Due to female infertility of SOD1(-/-) mice, SOD1(+/-) mice were selected for breeding and colony maintenance. SOD1 (+/-) knockout mice older than 8 weeks were selected as breeders, $10 \times 2 q$ per cage. After mating, as shown in Figure S1, SOD1(-/-) (homozygous), SOD1(+/-) (heterozygous), and SOD1 $(+/+)$ (wild type) were obtained. The genotyping of each mouse was assessed by quantitative PCR of DNA isolated from tail biopsy samples, as described on the Jackson Laboratory web site. Animals were divided in four groups: (1) normal diet (WT) group, (2) normal diet (SOD1-/-) group, (3) NAC-supplemented diet (SOD1/-) group, and (4) NAC-supplemented diet (WT) group. The antioxidant drug $\mathrm{N}$-acetylcysteine (NAC) was added to the drinking water at a concentration of $1 \mathrm{mg} / \mathrm{mL}$. The NAC concentration used in this study was similar to the concentration used in previous studies [22, 23].

2.2. Enzyme-Linked Immunosorbent Assay (ELISA). Mouse serum samples were collected. After pretreating collected containers with sodium heparin, we performed tail-cut blood collection of male mice at 2 months, 4 months, and 6 months. Samples were centrifuged at $3000 \mathrm{~g}$ for 25 minutes at $4^{\circ} \mathrm{C}$ to collect the upper serum. To determine the amount of lipid peroxidation in the serum, the MDA levels were analyzed spectrophotometrically using the modified thiobarbituric acid-reactive substance method as described by Zhou et al. [24]. The results were expressed as $\mathrm{nmol} / \mathrm{mL}$. The serum level of SOD, GSH-PX, and $\mathrm{H}_{2} \mathrm{O}_{2}$ were detected via specific kits 
(Jiancheng, Nanjing, China) following the manufacturer's instructions.

\subsection{Mandible Morphometric Analyses by Microcomputerized} Tomography (Micro-CT). Scans for volume, density, and bone mass of mandible alveolar bone were performed with a microtomograph imaging system $(\mu \mathrm{CT} 50$, Scanco Medical AG, Brüttisellen, Switzerland). The scanning parameters were X-ray bulb voltage $90 \mathrm{kV}$, current $200 \mu \mathrm{A}$, resolution $9 \mu \mathrm{m}$, and exposure time $500 \mathrm{~ms}$. The images were processed by three-dimensional reconstruction software ( $\mu$ CT Evaluation Program v6.0, Scanco Medical) and analyzed for determination of bone mass and density.

Bone mass analysis was performed on the alveolar bone of the first molar root bifurcation. The VOI (volume of interest) of the analysis region was selected as a cube with a length of $270 \mu \mathrm{m}$ below the alveolar crest of the left molar mandibular root bifurcation. The middle 30 layers were selected for trabecular bone parameter analysis, including bone mineral density (BMD), bone volume fraction (bone volume per total volume, BV/TV) (\%), trabecular thickness (Tb.Th), trabecular separation (Tb.Sp), and trabecular bone number (the mean trabecular number, Tb.N). Each sample was analyzed three times to calculate the average value.

2.4. Histology and TRAP Staining. Mandibles were removed, fixed in $4 \%$ paraformaldehyde overnight at $4^{\circ} \mathrm{C}$, and decalcified in $10 \%$ EDTA for 1 month at $37^{\circ} \mathrm{C}$. Decalcified mandibles were dehydrated and embedded in paraffin and cut at $5 \mu \mathrm{m}$ per slice on a rotary microtome. The sections were stained with alkaline tartrate-resistant acid phosphatase (TRAP) activity and immunohistochemically as described below. The number of osteoclasts per area, TRAP (+) cells $/ \mathrm{mm}^{2}$, was identified by the TRAP staining. All cells with 2 or more nuclei and close to trabeculae were considered osteoclastic cells. After applying TRAP solution (0.1 macetic acid buffer solution; naphthol AS-MX phosphate; fast red violet $\mathrm{LB}$ salt; $\mathrm{N}, \mathrm{N}$-dimethylformamide; Sigma-Aldrich Inc.), the sections were incubated at $37^{\circ} \mathrm{C}$ for $30 \mathrm{~min}$. A counterstaining with methyl green staining for 3 minutes was performed. The number of osteoclasts was measured using Image-Pro Plus 6.0 software (Rockville, MD). Three independent operators observed the sections and did the counting under the microscope.

2.5. Immunohistochemical Staining. Briefly, dewaxed and rehydrated paraffin-embedded sections were incubated with $3 \%(w / v)$ hydrogen peroxide for 10 minutes to block endogenous peroxidase activity and then washed twice in PBS. Antigen recovery was performed with pepsin antigen repair solution at room temperature for 10 minutes and then washed with PBS for 10 minutes. Blocking was completed with $5 \%(w / v)$ goat serum for 2 hours at room temperature. The slices were then incubated with the primary antibodies overnight at $4^{\circ} \mathrm{C}$. After washing with PBS, sections were incubated with secondary antibody (Sigma, St. Louis, MO, USA) for 30 minutes. After washing, brown pigmentation was likewise produced using 3,3-diaminobenzidine (DAB). Finally, the stained sections were counterstained with hematoxylin.
Images were acquired with a Leica microscope (Leica DM4000B, Solms, Germany) equipped with Leica software. Primary antibody included antibodies against Runx2 (ab102711; Abcam), alkaline phosphatase (ALP) (ab108337; Abcam), osteopontin (OPN) (ab8448; Abcam), and 4hydroxynonenal (4-HNE) (ab46545; Abcam) at a dilution of $1: 200$ according to the manufacturers' protocol. Approximately 6-8 sections were obtained from each sample of the same region. We used the Image-Pro Plus 6.0 software to compare the mean density (IOD/area) results with the same parameter settings for each sample.

2.6. Statistical Analysis. The data were analyzed using GraphPad Prism software (4.0) (San Diego, CA). Statistical analysis of data was performed using analysis of ANOVA and Student's $t$-test, followed by the Student-Newman-Keuls post hoc test for multiple group comparisons. Results are presented as mean \pm SD. Differences with a $P<0.05$ are considered statistically significant. All in vitro experiments were repeated at least three times.

\section{Results}

3.1. Mice Deficient in SOD1 (SOD1(-/-) Mice) Displayed Developmental Delays and Signs of Overall Oxidative Stress. Establishment of the SOD1 knockout mouse model was confirmed by successful deletion of SOD1 and total-SOD reduction in serum using ELISA (Figure 1(c)). At the same time, we measured the level of GSH-PX reductase in mice and found no obvious compensation with the decrease in SOD reductase (Figure $1(\mathrm{f})$ ), which meant that the defense ability of the enzyme antioxidant system against oxygen free radicals was dysfunctional. SOD1(-/-) mice exhibited lower weights and smaller body sizes compared to age-matched wild-type mice (Figures 1(a) and 1(b)), indicating that lack of SOD1 affected the growth and development of the whole body. SOD1(-/-) mice at 4-6 months exhibited a slight decrease in body weight, which might be related to mouse skeletal muscle atrophy [25].What is more, by detecting hydrogen peroxide and oxidative damage product MDA, we found that the level of serum $\mathrm{H}_{2} \mathrm{O}_{2}$ and MDA in the SOD1(-/-) mice were similar to those in the wild-type mice at 2 months of age $(P>0.05)$ (Figures $1(\mathrm{~d})$ and $1(\mathrm{e}))$. As mice aged, the levels of $\mathrm{H}_{2} \mathrm{O}_{2}$ and MDA in the SOD1(-/-) mice increased. The differences of serum $\mathrm{H}_{2} \mathrm{O}_{2}$ and MDA levels in SOD1(-/-) mice compared to controls were obvious at 4 months and 6 months $(P<0.05)$ (Figures $1(\mathrm{~d})$ and $1(\mathrm{e}))$. In conclusion, the accumulation of ROS due to the deficiency of SOD1 gradually appeared with age, ultimately leading to oxidative stress.

\subsection{Age-Related ROS Accumulation Caused Dual Effects on} Mandible Development. Three-dimensional scanning of SOD1(-/-) mice and the wild-type mandibles and analysis of the bone mass of alveolar bones were performed at 2 months, 4 months, and 6 months of the Figure 2. Previous studies reported that SOD1 gene knockout affected ovarian function, leading to abnormal secretion of follicle stimulating hormone (FSH), luteinizing hormone (LH), and progesterone (P) [26]. 


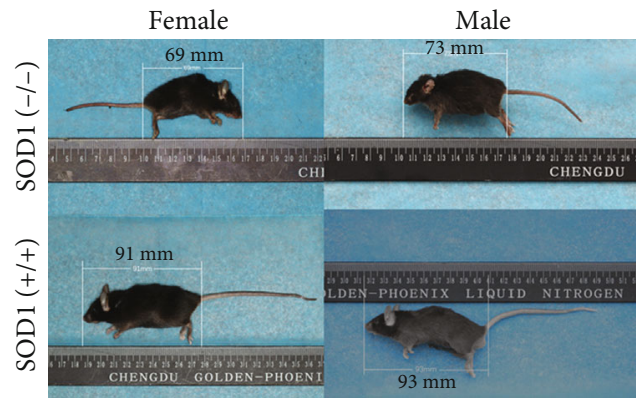

(a)
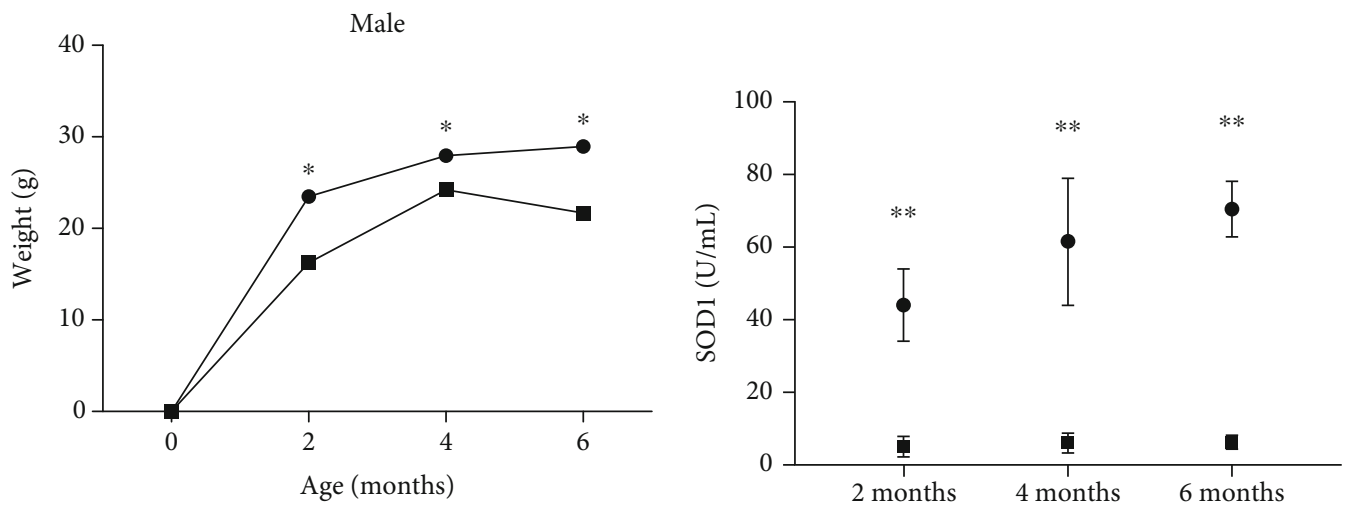

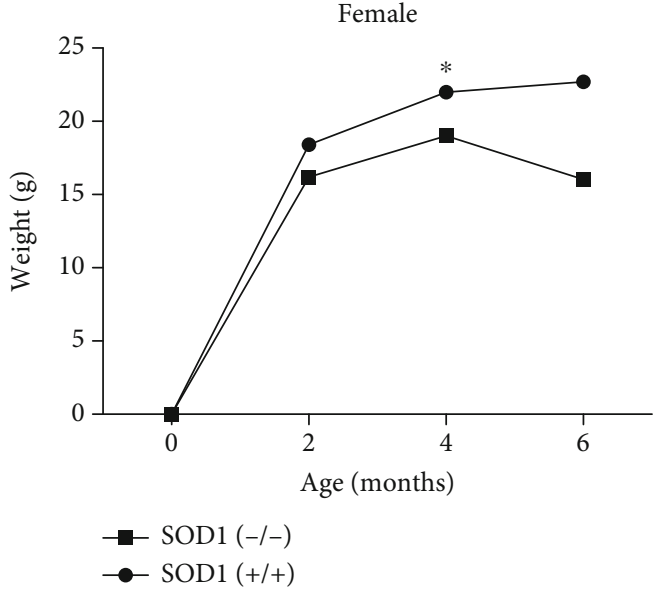

(b)

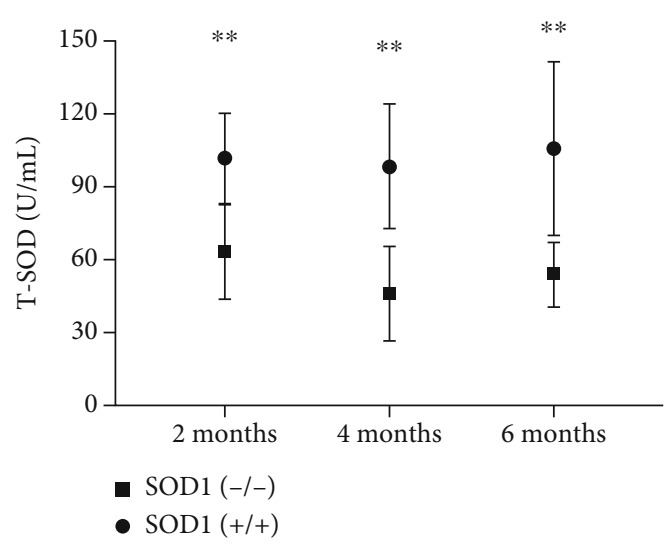

(c)

Figure 1: Continued. 


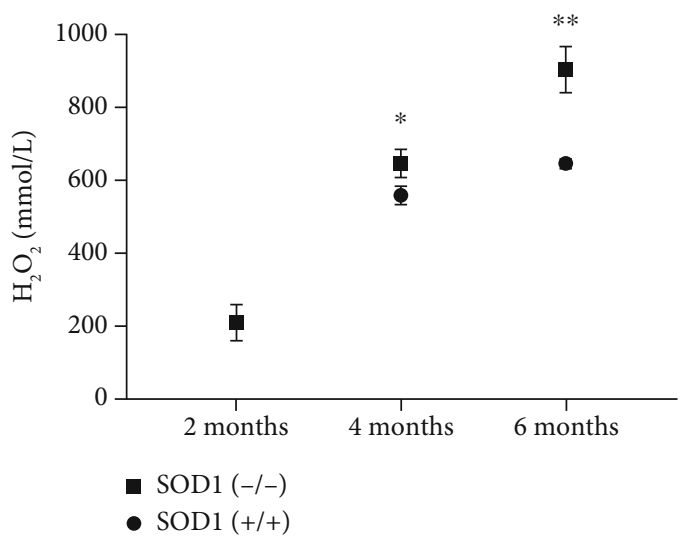

(d)

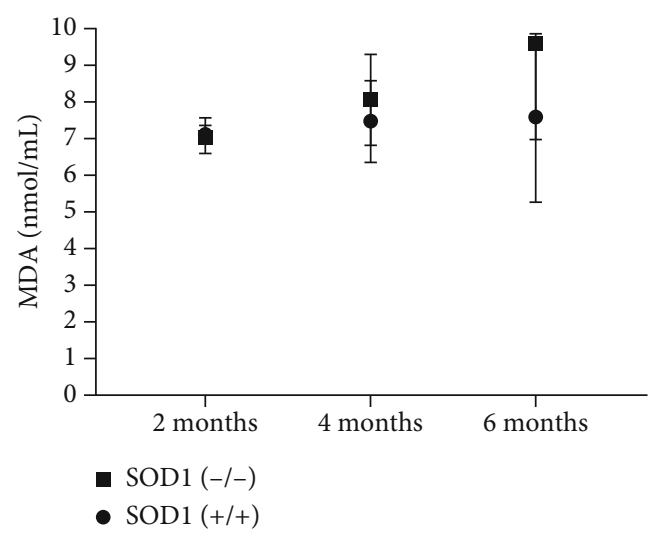

(e)

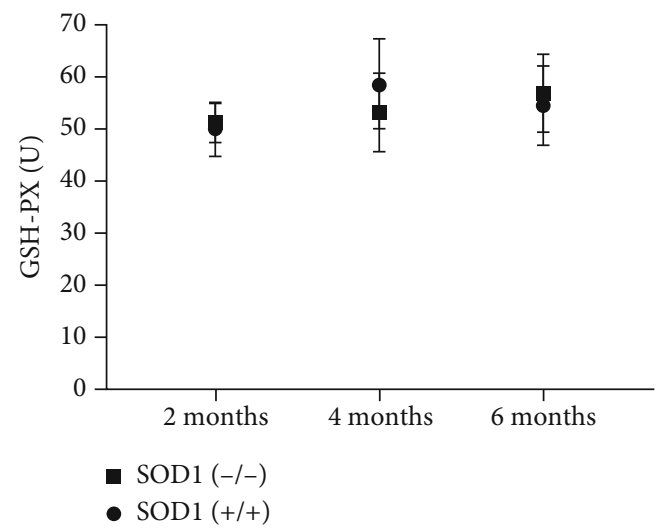

(f)

FIGURE 1: Analysis of oxidative stress state in SOD1(-/-) mice and wild-type mice. (a) SOD1(-/-) mice have smaller body size than agematched wild-type mice (3 months). (b) Body weight measurement of SOD1(-/-) mice and wild-type mice at different time periods. (c) Serum ELISA shows successful deleting of SOD1 and total-SOD reduction in SOD1(-/-) male mice. (d, e) Serum ELISA of $\mathrm{H}_{2} \mathrm{O}_{2}$ and oxidative damage product (MDA) show obvious ROS accumulation in SOD1(-/-) male mice. (f) Serum ELISA of GSH-PX reductase shows no difference between SOD1(-/-) male mice and wild-type mice. Date are shown as mean $\pm \operatorname{SD}(n=5) .{ }^{*} P<0.05$; ${ }^{* *} P<0.01$.

At the same time, these estrogens could affect the growth and development of the mandible [27], so male mice were used when analyzing the mandibular alveolar bone. Interestingly, the size of the 2-month-aged SOD1(-/-) mice's mandibular bone was larger than its counterpart in the wild-type mice. From 2 to 6 months of age, the mandible size of SOD1(-/-) mice did not change much while the wild-type mice showed progressive growth of mandibles. At 6 months of age, the mandible sizes of the SOD1(-/-) mice and the wild-type mice were basically similar. As shown in Figure 2, we did quantitative analysis of alveolar bone under the alveolar crest of the mandibular first molar root bifurcation area. At 2 months of age, bone mineral density (BMD), bone volume fraction (BV/TV), trabecular thickness (Tb.Th), trabecular bone separation (Tb.Sp), and trabecular bone number (Tb.N) in the SOD1(-/-) mice were not statistically significantly different compared with the wild-type mice. At 4 months of age, compared with the wild-type mice, the bone volume fraction and trabecular thickness of the male SOD1(-/-) mice increased (Figures 2(d) and 2(e)), and the trabecular bone separation decreased $(P<0.05)$ (Figure 2(f)). At 6 months of age, the results were opposite. Compared with the wild-type mice, the bone mineral density and bone volume fraction of the male SOD1(-/-) mice were reduced (Figure 2(d)), and the trabecular bone separation was increased $(P<0.05)$ (Figure 2(f)).

3.3. Low Osteoclast Amount and Mutative Osteogenic Associated Protein Expression in an Age-Dependent Manner Were Observed in SOD1(-/-) Mice. In healthy bone tissue, a delicate homeostasis is maintained between the action of the osteoclasts in bone resorption and the action of osteoblasts in bone formation. We observed the number of osteoclasts through TRAP staining in the alveolar crest of the mandibular first molar root bifurcation area (Figure 3(a)). In both wild-type mice and SOD1(-/-) mice, there were almost no TRAP-positive osteoclasts in the alveolar bone at 2 months of age. With increasing age, the number of osteoclasts increased in the wild-type mice. In the SOD1(-/-) mice, the counts of osteoclasts increased, yet at a lower amount compared to wild-type mice. At 6 months of age, the number of TRAP-positive osteoclasts in SOD1(-/-) mice was significantly lower than that in the wild-type mice $(P<0.01)$, indicating that SOD1 deficiency impaired bone resorption. At the same time, we also performed immunohistochemistry of the 


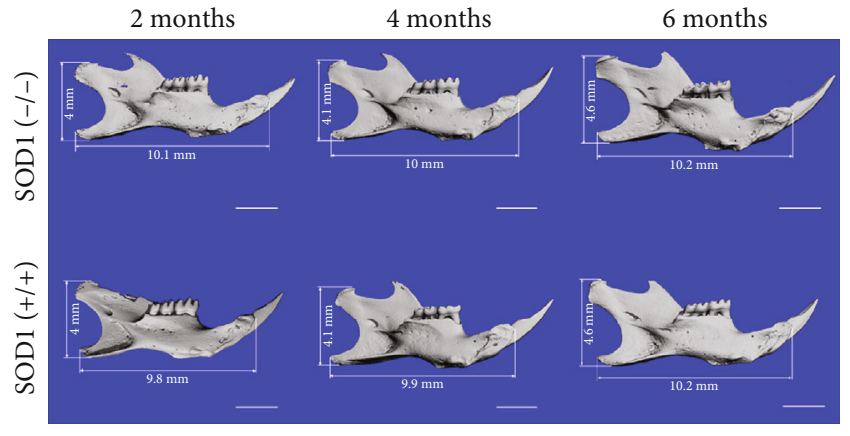

(a)

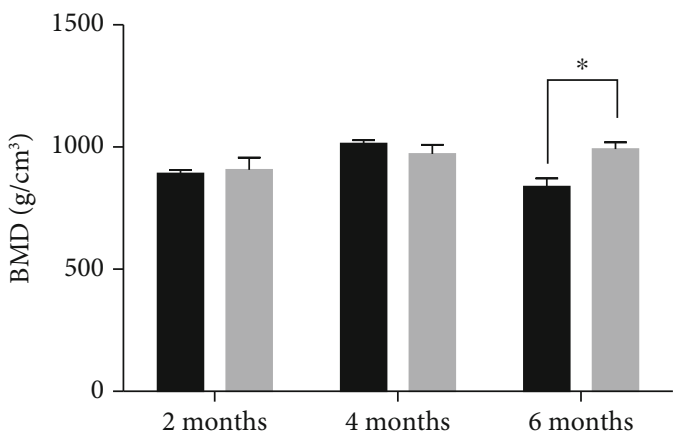

COD1 (-/-)

SOD1 $(+/+)$

(c)

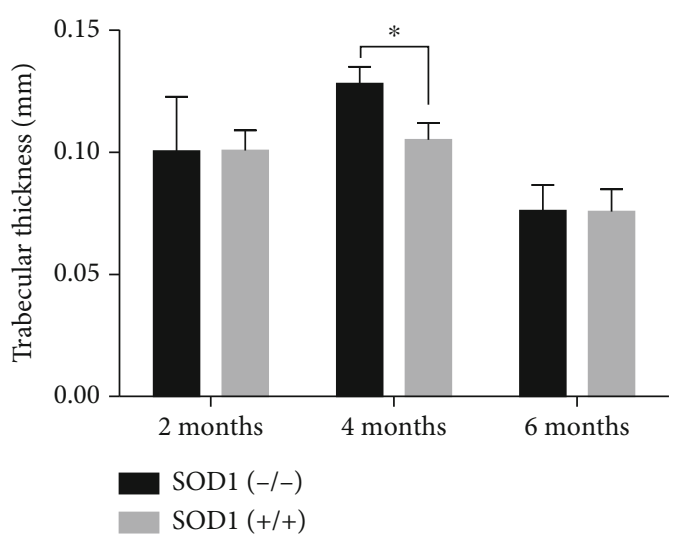

(e)

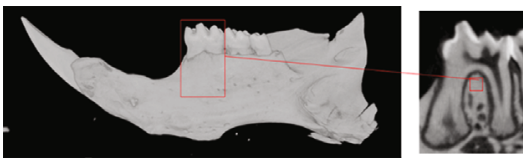

(b)

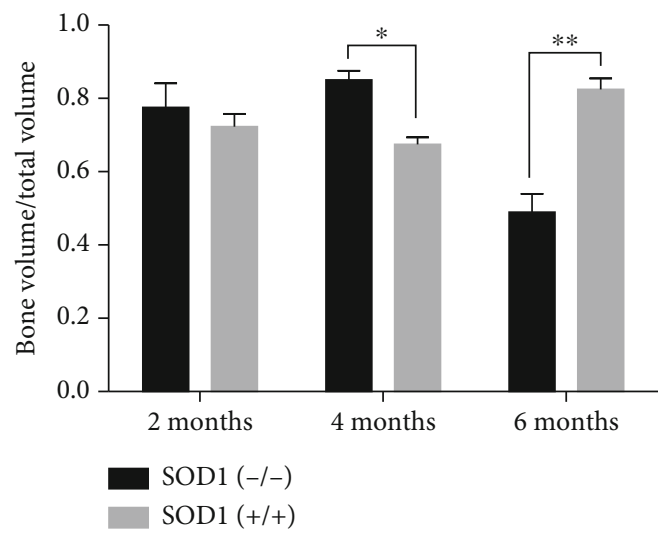

(d)

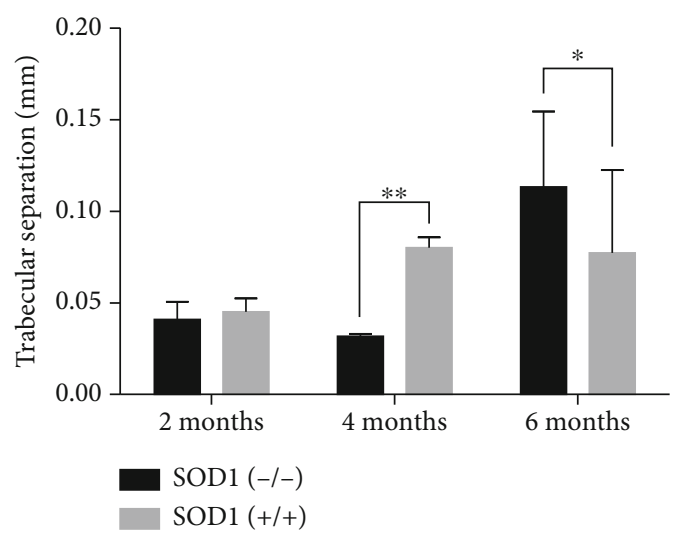

(f)

Figure 2: Continued. 


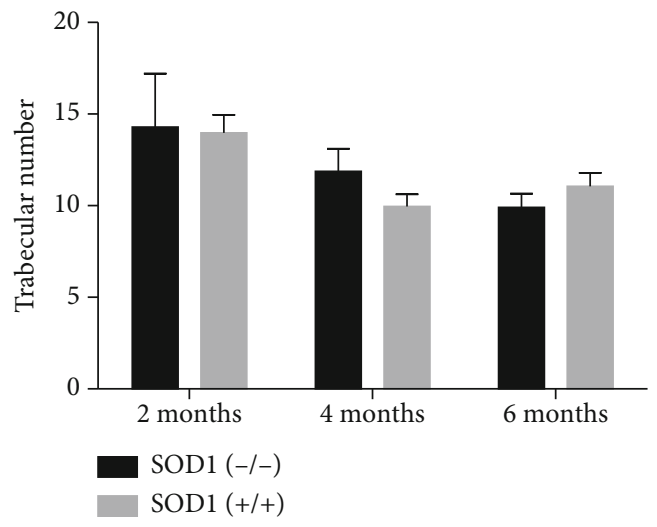

(g)

Figure 2: Morphological characteristics and micro-CT of the mandibles in SOD1(-/-) male mice and wild-type male mice. (a) Microcomputed tomography reconstruction of mandibles at 2 months, 4 months, and 6 months. (b) Quantitative analysis of alveolar bone under the alveolar crest of the mandibular first molar root bifurcation area. (c-g) Bone mineral density (BMD), bone volume fraction (BV/TV), trabecular thickness (Tb.Th), trabecular bone separation (Tb.Sp), and trabecular bone number (Tb.N) of alveolar bone in first molar root bifurcation area. Data are shown as mean $\pm \mathrm{SD}(n=5) .{ }^{*} P<0.05 ;{ }^{* *} P<0.01$. Scale bars (a) $2 \mathrm{~mm}$.

transcription factor Runx2 and osteogenesis markers OPN and ALP in mandibles (Figure 3 ). In earlier ages (2 months), the expression levels of Runx2 and OPN in the SOD1(-/-) mice were significantly higher than those in the wild-type mice (Figures 3(g) and 3(h)). At 4 months, the expression levels of Runx2, OPN, and ALP remained higher compared to the wild-type mice. Notably, a sharp reduction of Runx2 and OPN could be found at the age of 6 months in the SOD1(-/-) mice (Figures 3(c) and 3(d)), indicating that the ability of alveolar bone formation in SOD1 knockout mice also increased at first and then decreased over time. Generally, 4-HNE, a biofilm lipid peroxidation product, can reflect the degree of oxidative damage in the alveolar bone region [28]. Immunohistochemistry of 4 -HNE in alveolar bone showed that the oxidative stress of the jaw had been becoming more and more obvious with age in SOD1(-/-) mice (as shown in Figure S2).

3.4. Antioxidant NAC Application Reversed Oxidative Stress and Caused Low ROS Concentration in SOD1(-/-) Mice. One of the most commonly used antioxidants is $\mathrm{N}$ acetylcysteine (NAC), which is the precursor of reduced glutathione (GSH) in cells [29]. Increasing the content of NAC can promote the synthesis of GSH-PX and help remove the excess active oxygen free radicals [30, 31]. After adding antioxidant NAC to the diet of mice, the MDA levels in serum and 4-HNE levels in alveolar bone of the SOD1(-/-) mice showed significant decline compared with those in the normal feeding group $(P<0.01)$ (Figure $4(\mathrm{a})$ and Figure S3). More importantly, under normal feeding conditions, SOD1(-/-) mice had higher serum MDA levels and 4-HNE levels in alveolar bone than wild-type mice, but the difference was eliminated after adding NAC. Interestingly, the serum MDA content and 4-HNE levels in alveolar bone of the SOD1(-/-) mice feeding with NAC were even lower than the wild-type mice on normal diet and did not change significantly with age and remained below physiological levels (Figure 4(a) and Figure S3). Moreover, after adding antioxidant NAC in the diet of mice, serum GSH-PX levels in the SOD1(-/-) mice and the wildtype mice showed no significant difference between each other $(P>0.05)$, whereas, compared with normal diet groups, both SOD1(-/-) mice and wild-type mice exhibited higher levels of GSH-PX after adding NAC $(P<0.01)$ (Figure $4(\mathrm{~b}))$.

\subsection{Effects of NAC-Induced Decrease in ROS Concentration} on Jaw Formation and Bone Metabolism of SOD1(-/-) Mice. Micro-CT analysis showed that the mandibular size of the SOD1(-/-) mice after 2 months of NAC feeding (4 months old) was larger than that of the normal feeding group (Figure 4(c)). After 4 months (6 months old), we found that BMD, BV/TV, Tb.Th, and Tb.N increased in the SOD1(-/-) NAC group, compared with the SOD1(-/-) normal feeding group. No significant differences were found in BMD and bone mass $(\mathrm{BV} / \mathrm{TV})$ between the normal wild-type group and the SOD1(-/-) NAC group $(P>0.05)$ (Figures $4(\mathrm{e})$ and $4(\mathrm{f}))$. It is noteworthy that BMD, BV/TV, and Tb.Th decreased after adding NAC in the diet in the wild-type mice at 4 months old $(P<0.05)$ (Figures $4(\mathrm{e})$ and $4(\mathrm{f}))$. The number of osteoclasts in both the SOD1(-/-) group and the wildtype group was significantly reduced after adding NAC in the diet $(P<0.05)$ (Figure 5(a)). Although ROS aggregation had been reversed and ROS concentration had shrunk, the alveolar bone remodeling activity was maintained at a low level. As for the bone formation, comparing the wild-type NAC group with the wild-type normal feeding group, we found that Runx 2 and OPN did not change significantly at 2 months (4 months old), while decreases were observed at 4 months after the dosing (6 months old) (Figures 5(c) and 5(d)), indicating that reduction of ROS concentration to a certain extent would lead to weaker osteoblast activity. At 6 months old, SOD1(-/-) mice exhibited obvious bone loss. We found that the use of NAC significantly increased the expression of ALP and Runx2 (Figures 5(f) and 5(g)). 


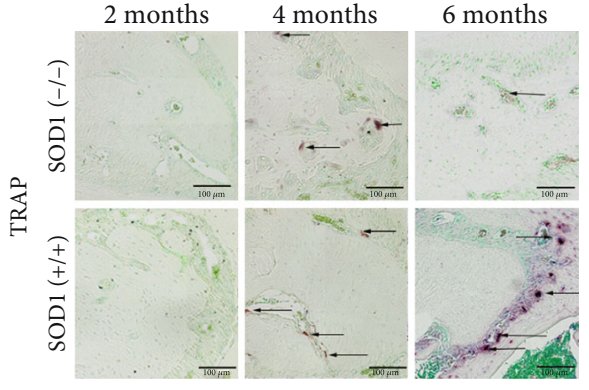

(a)
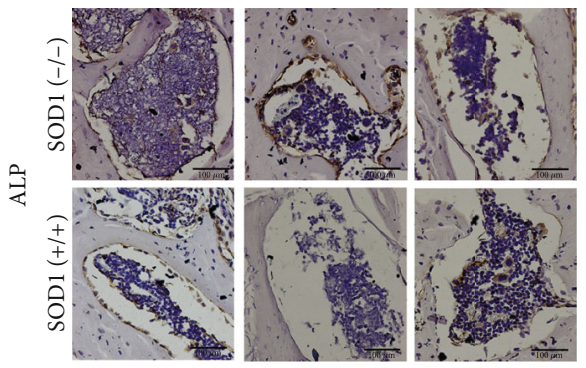

(b)

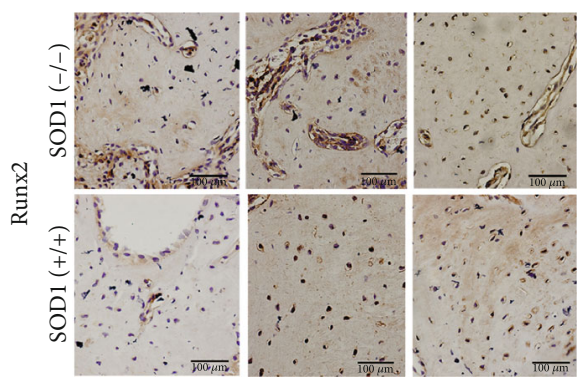

(c)

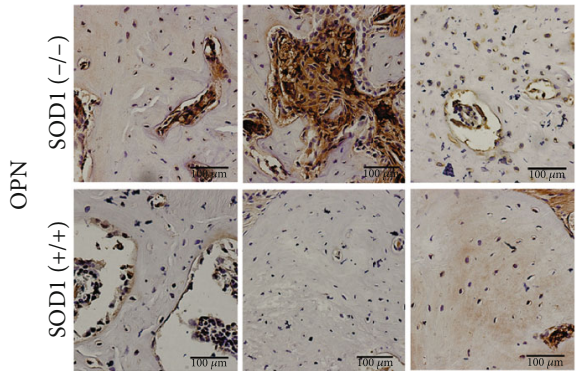

(d)

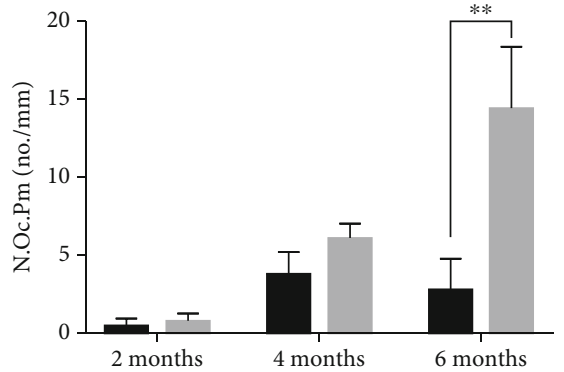

(e)

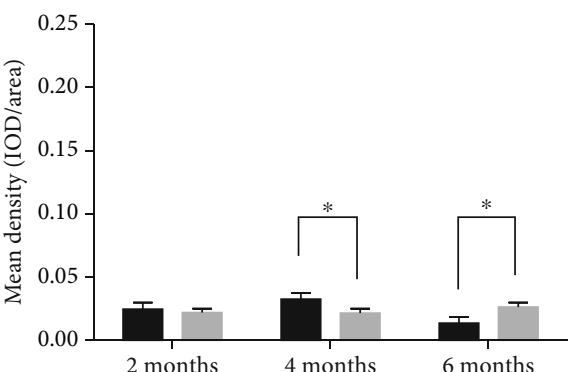

(f)

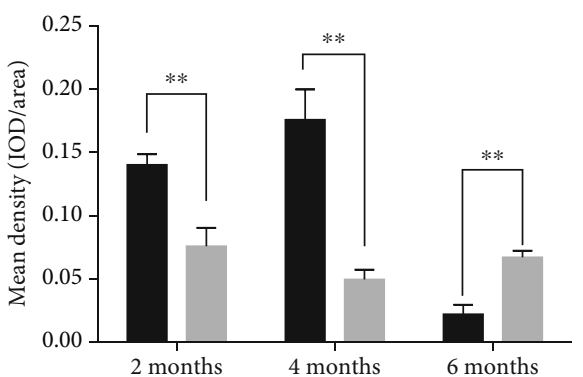

(g)

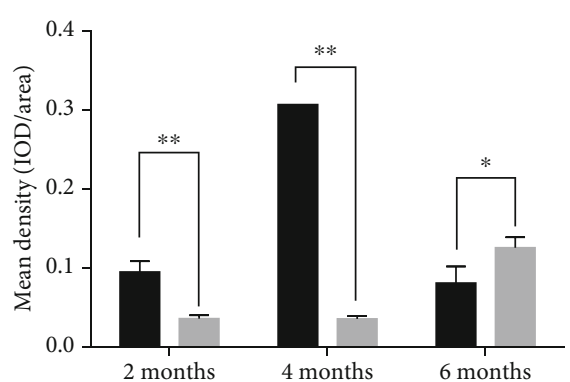

(h)

SOD1 $(-/-)$

SOD1 $(+/+)$

FIgURE 3: Bone metabolism of the mandible in SOD1(-/-) male mice and wild-type male mice. (a) TRAP staining shows lower number of osteoclasts in alveolar bone under the alveolar crest of the mandibular first molar root bifurcation area of SOD1(-/-) mice. Arrows indicate active osteoclasts. (b) Immunohistochemistry of ALP in the same place. (c) Immunohistochemistry of Runx2 in the same place. (d) Immunohistochemistry of OPN in the same place. $(\mathrm{e}-\mathrm{h})$ Quantitative analysis of $(\mathrm{a}-\mathrm{d})$, respectively. Data are shown as mean \pm SD $(n=5)$. ${ }^{*} P<0.05 ;{ }^{* *} P<0.01$. Scale bars (a-d) $100 \mu \mathrm{m}$.

\section{Discussion}

Since Denham Harman proposed the free radical doctrine in 1956, many degenerative diseases such as heart disease, Alz- heimer's disease, Parkinson's disease, and tumors have been shown to be linked to excessive free radicals [32-34]. Through signaling, regulating stem cell fate, and immunity, ROS play an important role in maintaining homeostasis 


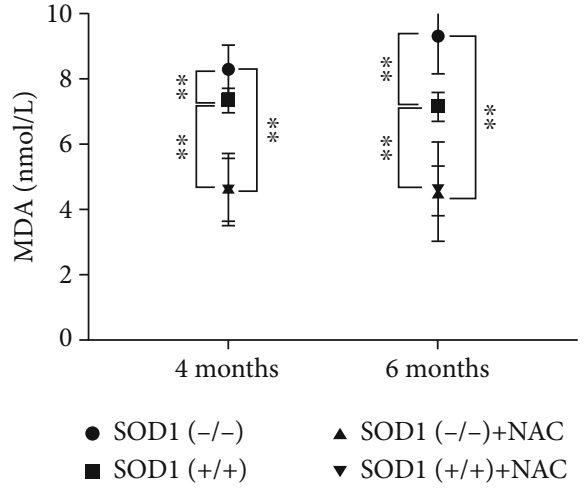

(a)

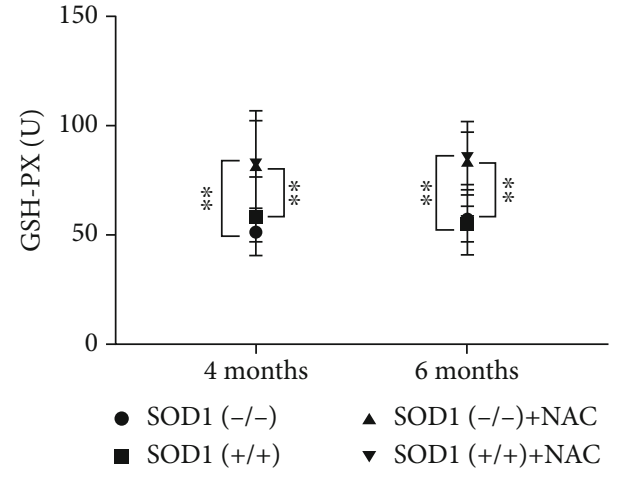

(b)

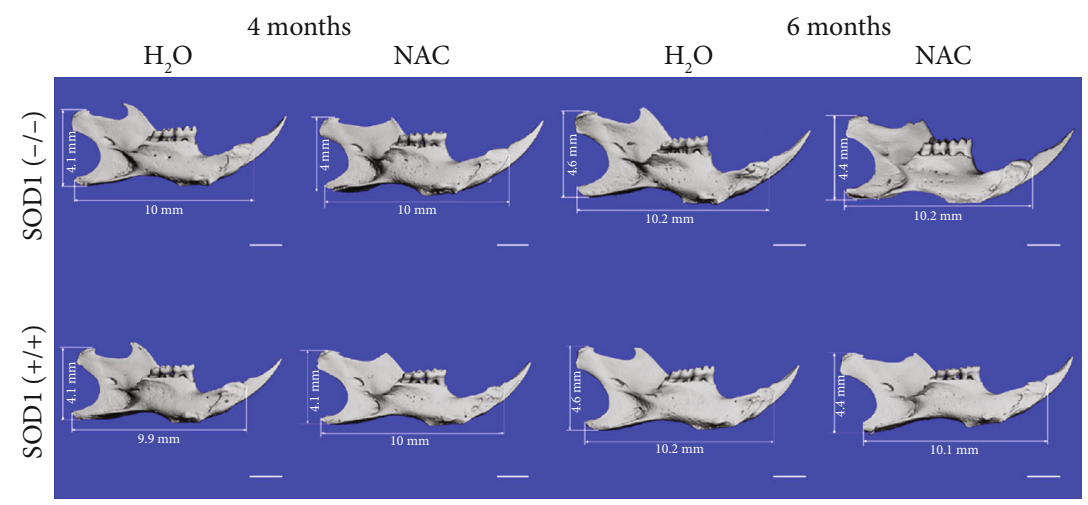

(c)

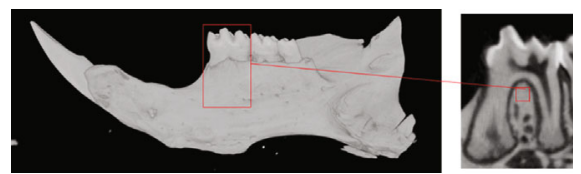

(d)

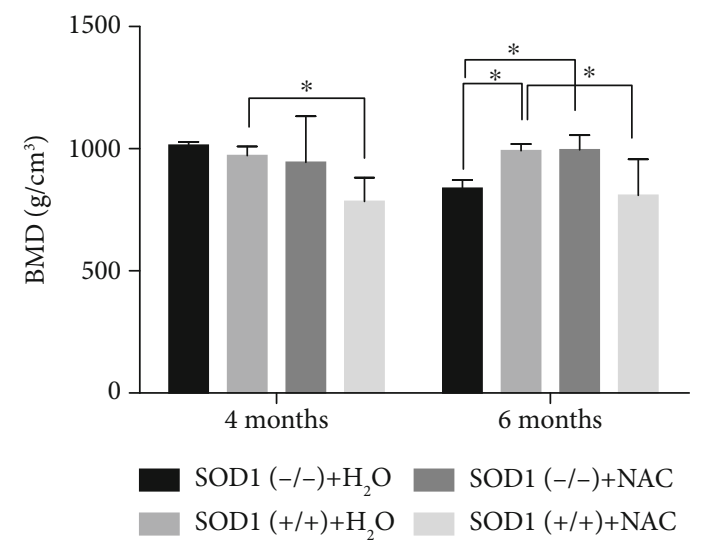

(e)

FIgURE 4: Continued. 


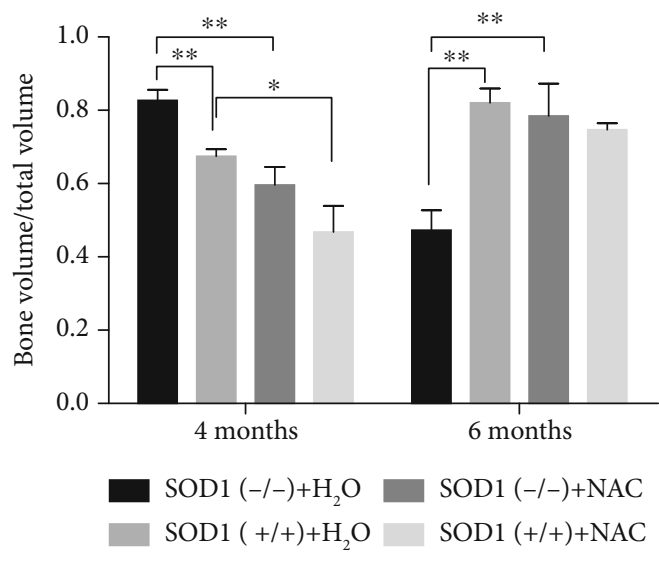

(f)

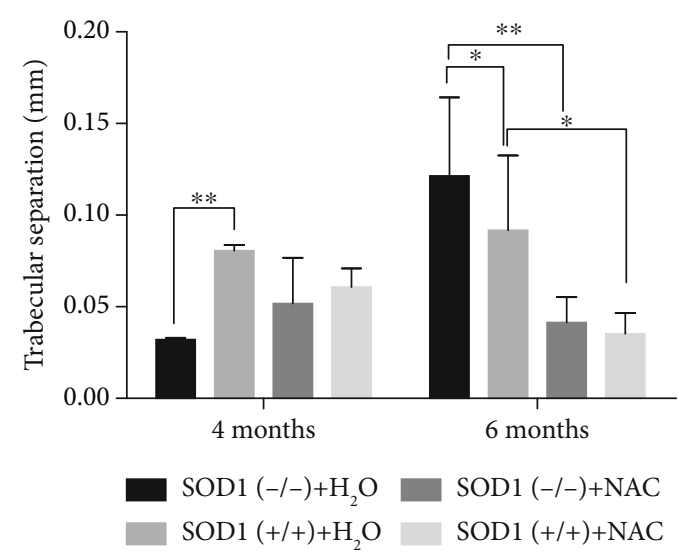

(h)

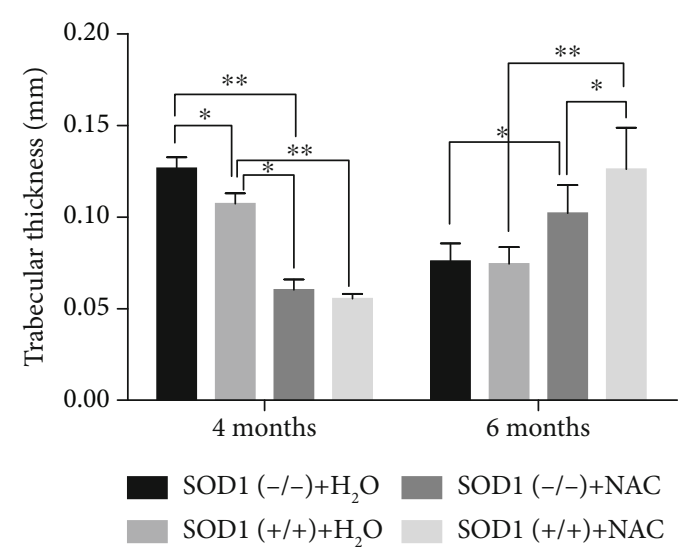

(g)

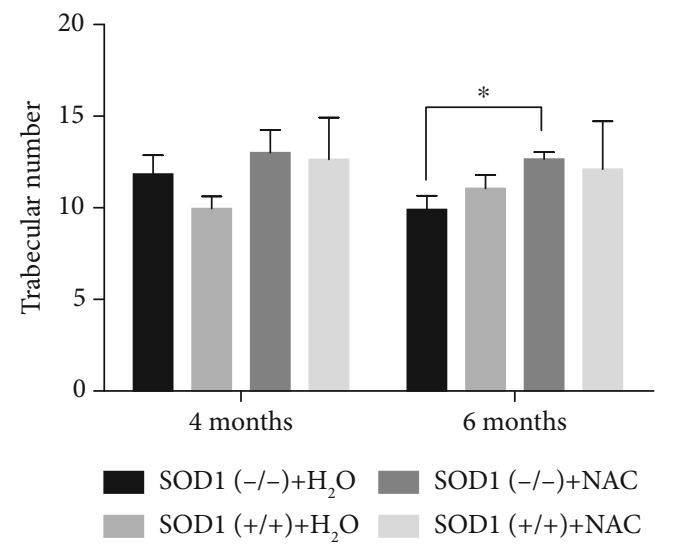

(i)

Figure 4: Antioxidant NAC application reverse oxidative stress and affect mandible formation. (a) ELISA shows lower MDA level in serum after adding antioxidant NAC in the diet of mice. (b) ELISA shows higher GSH-PX level in serum after adding antioxidant NAC in the diet of mice. (c) Microcomputed tomography reconstruction of mandibles of SOD1(-/-) male mice after feeding with NAC. (d) Quantitative analysis of alveolar bone under the alveolar crest of the mandibular first molar root bifurcation area. (e-i) Bone mineral density (BMD), bone volume fraction (BV/TV), trabecular thickness (Tb.Th), trabecular bone separation (Tb.Sp), and trabecular bone number (Tb.N) of alveolar bone in first molar root bifurcation area. Data are shown as mean $\pm \mathrm{SD}(n=5) .{ }^{*} P<0.05 ;{ }^{* *} P<0.01$. Scale bars (c) $2 \mathrm{~mm}$.

and promoting normal development [35], but the specific role in various tissues and organs has not yet been proven definitively.

Previous studies have shown that ROS accumulation caused by SOD1 dysregulation can lead to increased bone mass reduction, skeletal fragility, and low-turnover osteoporosis in long bones $[18,36]$. In the current study, we demonstrated for the first time that increased ROS levels caused by SOD1 deficiency resulted in enlargement of mandible size and increased bone mass at early age (2-4 months). In contrast, mandibles of SOD1(-/-) mice at older age (6 months) exhibited a significant reduction in bone mass. These data suggest that ROS aggregation has dual effects on mandibular alveolar bone: promotion or pathogenic effect, which depends on ROS levels and stages of development. Notably, the phenotypes of long bones and mandibles were not consistent despite the same age of SOD1(-/-) mice (4 months). Nojiri et al. [18] found decreases in total bone density, BV/TV, Tb.N, and Tb.Th and an increase in trabecular separation in the femur of SOD1(-/-) mice. Our results in man- dibular alveolar bones demonstrated the opposite, suggesting that different types of bones have different responses and tolerances to ROS changes. Mandibular alveolar bones and long bones have different developmental origins and responses. Mandibles originate from the neural crest of the ectoderm, while mesenchymal cells of the mesoderm develop into long bones. Previous studies have found that bone marrow mesenchymal stem cells derived from rat mandibles had significantly higher proliferation capacity and mineralization ability in vitro than bone marrow mesenchymal stem cells derived from long bones [37]. Moreover, in some diseases, such as osteoporosis and hyperthyroidism, the degree of involvement in long bones is significantly higher than that in the jaws [38].

In the early stages of mandible development (2 months), the cranial and maxillofacial regions of C57 mice generally grow most rapidly (close to $80 \%$ of the growth is completed) [39]. Although overall ROS levels are not elevated and oxidative stress is low, cytoplasmic and mitochondrial ROS may be slightly elevated [40]. Therefore, enlargement of mandible 


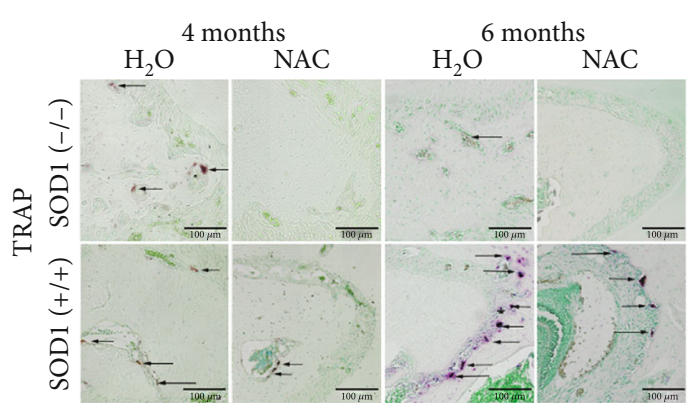

(a)

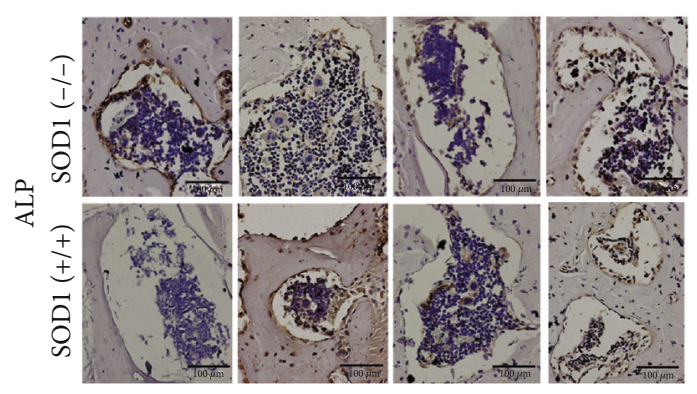

(b)

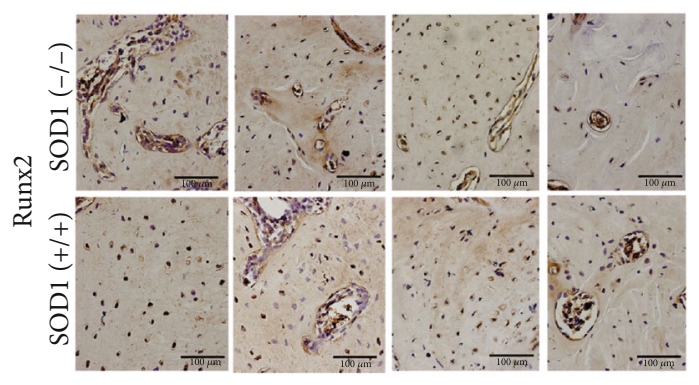

(c)

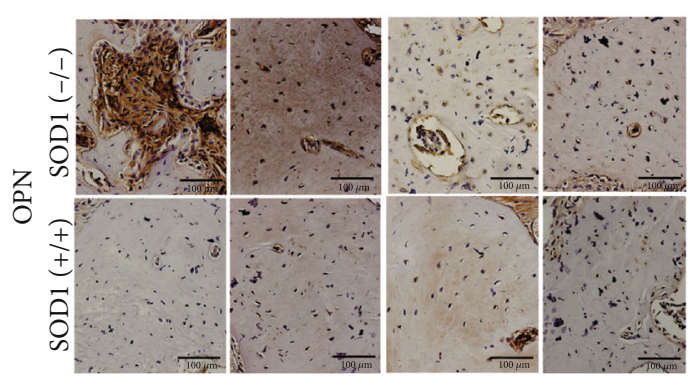

(d)

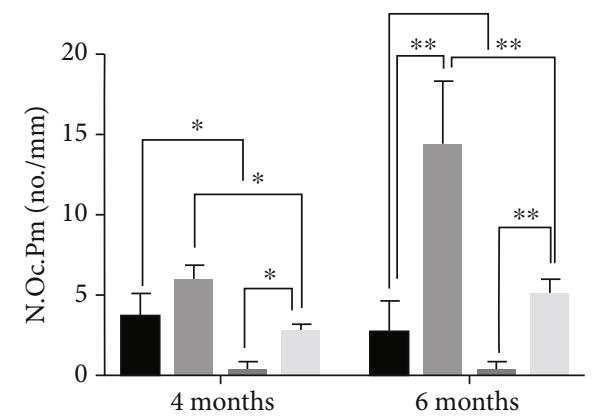

(e)

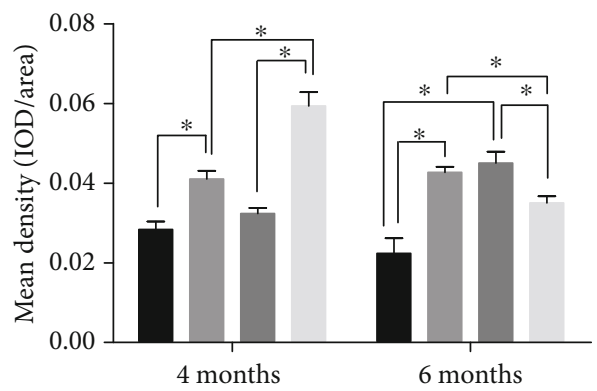

(f)

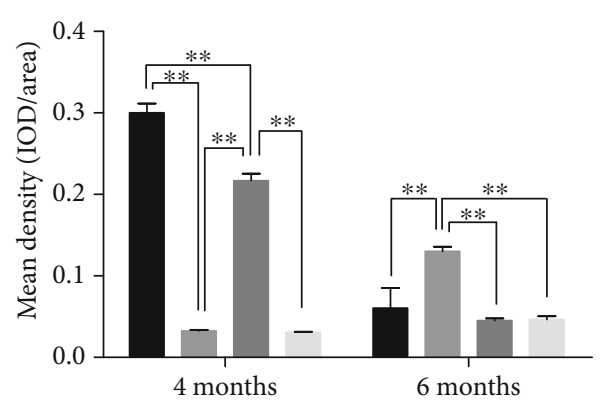

(g)

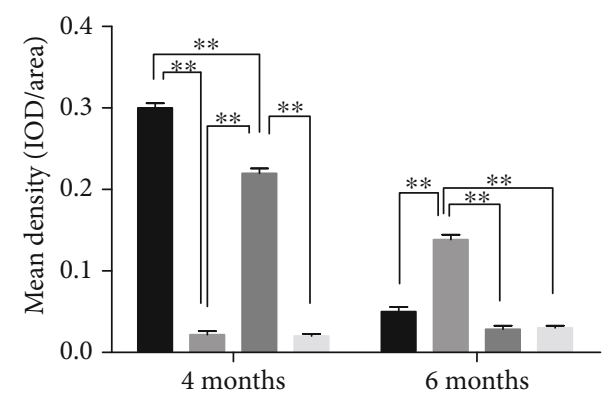

(h)

SOD1 $(-/-)+\mathrm{H}_{2} \mathrm{O} \square \operatorname{SOD} 1(-/-)+\mathrm{NAC}$ SOD1 $(+/+)+\mathrm{H}_{2} \mathrm{O} \quad$ SOD1 $(+/+)+\mathrm{NAC}$

FIgURE 5: Antioxidant NAC application affect bone metabolism. (a) TRAP staining shows lower number of osteoclasts after adding NAC in a diet of mice. Arrows indicate active osteoclasts. (b) Immunohistochemistry of Runx2. (c) Immunohistochemistry of OPN. (d) Immunohistochemistry of ALP. (e-h) Quantitative analysis of $(\mathrm{a}-\mathrm{d})$, respectively. Data are shown as mean \pm SD $(n=5)$. ${ }^{*} P<0.05 ;{ }^{* *} P<$ 0.01. Scale bars $(\mathrm{a}-\mathrm{d}) 100 \mu \mathrm{m}$.

size may result from $\mathrm{H}_{2} \mathrm{O}_{2}$ slowly accumulating in the cell, exerting its role as a signal molecule, and promoting the proliferation and differentiation of bone marrow stem cells in the initial stage of SOD1 deficiency [41]. With the increase of age
(4 months), we found higher expression of osteogenesisrelated proteins (Runx2, ALP, and OPN) and fewer osteoclasts in mandibles of SOD1(-/-) mice (Figure 3$)$. At the same time, we also found that SOD1(-/-) mice mandibles had 
higher levels of 4-HNE than those of the wild-type mice. Interestingly, previous studies showed that 4-HNE can act as a signaling molecule to promote cell proliferation and affect transcription at a certain concentration [42]. Increased expression of ALP, OPN, and Runx 2 may be associated with mild elevated $\mathrm{H}_{2} \mathrm{O}_{2}$ and 4-HNE levels in mandibular alveolar bones of the SOD1(-/-) mice. These results indicated that the increase of ROS may promote the mineralization of the bone matrix and the deposition of calcium when the development of the mandibular alveolar bone is basically stable. At the same time, elevated ROS level may reduce bone resorption, thereby increasing the bone mass and density of the mandibular alveolar bone.

When ROS continued to accumulate, at 6 months of age, the mandibular alveolar bone showed obvious oxidative stress, decreased BMD and bone mass, and decreased ALP, OPN, and Runx2 expressions (Figures 2 and 3). Despite the obvious bone loss, our results showed that the number of TRAP-positive osteoclasts reduced, which support the idea that accumulated ROS may have resulted in the suppression of osteoclastogenesis and low-turnover bone loss [18]. Another possibility is increased ROSinduced ROS release (RIRR), which means that elevated ROS function through diminishing the efficacy of antioxidant defenses and inducing the activation of transcription factors of ROS-producing enzymes to increase ROS production [43-45]. The rapid expansion of ROS may cause severe oxidative stress in jaw bones, inducing apoptosis and autophagy [46,47], which damages the function and reduces the number of osteoblasts and osteoclasts, thereby destroying bone homeostasis and contributing to bone diseases.

Considering the concentration-dependent effect of ROS [48], controlling ROS in the appropriate range is a potential treatment. Given that SOD reductase is easily metabolized after entering the body for the poor tissue affinity and instability [49], we use the antioxidant NAC, the precursor of reduced glutathione (GSH) in the cell, to help clear excess reactive oxygen radicals. In our study, we demonstrated that $\mathrm{N}$-acetylcysteine could reverse the pathogenic impact of ROS aggregation on the mandibular alveolar bone: improving bone formation and increasing bone mass. The increase in the content of NAC can promote the synthesis of GSH (Figure 4) and help remove excess reactive oxygen species accumulated in the body [50]. Balszuweit et al. [32] also reported that NAC protected against LPS-induced inflammatory bone loss via reducing ROS formation in macrophages and osteoclasts. Mandibular alveolar bone oxidative stress level in SOD1(-/-) mice, displayed by levels of 4-HNE and MDA, was effectively alleviated, even lower than normal condition after the addition of antioxidant NAC (Figure 4), which suggested it also could be used as a model to study the effect of ROS reduction on mandibular alveolar formation. Our results showed that SOD1(-/-) mice have significantly improved BMD, BV/TV, Tb.Th, and Tb.N of mandibular alveolar bone after taking NAC for 4 months (6 months old), indicating that superoxide-induced bone loss could be improved by antioxidant treatment. Additionally, NAC treatment also produced increased osteogenesis- related protein expression (ALP, Runx2, and OPN) and decreased number of osteoclasts. However, in the wild-type mice at 6 months, the use of NAC led to reduced BMD, bone mass, and expression of ALP, Runx2, and OPN. These results imply that NAC might protect against bone loss via reducing ROS formation in macrophages and osteoclasts [51]. The reduction of ROS caused by antioxidants, even lower than normal physiological concentration, may inhibit osteogenesis. Previous studies showed that excessively low levels of ROS can also cause pathological changes by disturbing the physiological role of oxidants in proliferation, vasodilation, and host defense and may lead to cancer development [52]. Therefore, excessively low ROS concentration is not conducive to mandible development. Together with our results, these reports suggest that NAC could represent a candidate for the treatment of bone loss in patients with oxidative stress. However, given the concentration-dependent effects of ROS, we do not recommend prophylactic, prolonged, large-dose use of antioxidants [53]. We have not observed significant changes in the cementum of SOD1(-/-) mice (Figure S4). However, whether the alveolar bone changes of SOD1(-/-) mice will affect the periodontal ligament attached to it and the related mechanisms await further study.

In conclusion, this study showed the dual effects of ROS aggregation on mandibular alveolar bone formation caused by SOD1 deficiency: bone mass (BV/TV, Tb.Th) and expression of osteogenic related proteins (ALP, Runx2, and OPN) increased first and then decreased compared with wild-type mice. This is likely related to the ROS level of the mandibular alveolar bone and the age of the mice. Different concentrations of ROS and even oxidation products (4-HNE) serve as signal molecules, participating in the redox signal network to regulate the normal physiological and pathological state of the body, which is critical in redox biology [54]. We expect that additional exploration in molecular effectors of redox biology in the future will lead to therapies that selectively reduce disease without disturbing healthy tissues.

\section{Data Availability}

The data used to support the findings of this study are available from the corresponding author upon request.

\section{Ethical Approval}

The animal study was reviewed and approved by the Ethics Committee of the State Key Laboratory of Oral Diseases, West China Hospital of Stomatology (Chengdu, Sichuan) (license number WCHSIRB-D-2016-195).

\section{Conflicts of Interest}

The authors declare that the research was conducted in the absence of any commercial or financial relationships that could be construed as a potential conflict of interest. 


\section{Authors' Contributions}

YZ, YY, and WG contributed to the conception, design, data acquisition, analysis, and interpretation; drafted; and critically revised the manuscript; MX, JZ, and YX contributed to the data acquisition and interpretation and critically revised the manuscript; and GC, QG, and WT contributed to the data analysis and critically revised the manuscript. All authors gave final approval and agree to be accountable for all aspects of the work. Yunyan Zhang and Yuzhi Yang contributed equally to this work.

\section{Acknowledgments}

We are grateful to the Animal Experiment Center of Sichuan University for providing help for mice reproduction. This work was supported by the Nature Science Foundation of China (31771062, 31971281), National Key Research and Development Program of China (No. 2017YFA0104800), and Key Research and Development Program of Sichuan Province (2017SZ0031).

\section{Supplementary Materials}

Figure S1: quantitative PCR analysis of DNA of SOD1(-/-) mice, SOD1(+/-) mice, and SOD1(+/+) mice. Figure S2: immunohistochemistry of 4-HNE in alveolar bone under the alveolar crest of the mandibular first molar root bifurcation area of SOD1(-/-) male mice and wild-type male mice and associated quantitative analysis. Data are shown as mean \pm SD $(n=5) .{ }^{*} P<0.05 ;{ }^{*} P<0.01$. Figure S3: immunohistochemistry of 4-HNE in alveolar bone under the alveolar crest of the mandibular first molar root bifurcation area of SOD1(-/-) male mice and wild-type male mice after adding NAC in diet and associated quantitative analysis. Data are shown as mean $\pm \mathrm{SD}(n=5) .{ }^{*} P<0.05 ;{ }^{* *} P<0.01$. Figure S4: HE staining of alveolar bone under the alveolar crest of the mandibular first molar root bifurcation area of SOD1(-/-) male mice and wild-type male mice. D: dentin; PDL: periodontal ligament; C: cementum. Cementum and dentin are distinguished by white dotted lines. Scale bars: $200 \mu \mathrm{m}$ and $20 \mu \mathrm{m}$. (Supplementary Materials)

\section{References}

[1] B. Perillo, M. Di Donato, A. Pezone et al., "ROS in cancer therapy: the bright side of the moon," Experimental \& Molecular Medicine, vol. 52, no. 2, pp. 192-203, 2020.

[2] Y. F. Chen, H. Liu, X. J. Luo et al., "The roles of reactive oxygen species (ROS) and autophagy in the survival and death of leukemia cells," Critical Reviews in Oncology/Hematology, vol. 112, pp. 21-30, 2017.

[3] S. A. Syed Mortadza, L. Wang, D. L. Li, and L. H. Jiang, "TRPM2 channel-mediated ROS-sensitive Ca2+ signaling mechanisms in immune cells," Front Immunol, vol. 6, no. $407,2015$.

[4] C. R. Reczek and N. S. Chandel, "ROS-dependent signal transduction," Current Opinion in Cell Biology, vol. 33, pp. 8-13, 2015.
[5] I. Liguori, G. Russo, F. Curcio et al., "Oxidative stress, aging, and diseases," Clinical Interventions in Aging, vol. 13, pp. 757-772, 2018.

[6] A. Okado-Matsumoto and I. Fridovich, "Subcellular distribution of superoxide dismutases (SOD) in rat Liver," The Journal of Biological Chemistry, vol. 276, no. 42, pp. 38388-38393, 2001.

[7] L. Banci, L. Barbieri, I. Bertini et al., "Atomic-resolution monitoring of protein maturation in live human cells by NMR," Nature Chemical Biology, vol. 9, no. 5, pp. 297-299, 2013.

[8] L. Pouyet and A. Carrier, "Mutant mouse models of oxidative stress," Transgenic Research, vol. 19, no. 2, pp. 155-164, 2010.

[9] D. Jaarsma, F. Rognoni, W. van Duijn, H. W. Verspaget, E. D. Haasdijk, and J. C. Holstege, "CuZn superoxide dismutase (SOD1) accumulates in vacuolated mitochondria in transgenic mice expressing amyotrophic lateral sclerosis-linked SOD1 mutations," Acta Neuropathologica, vol. 102, no. 4, pp. 293305, 2001.

[10] K. Hashizume, M. Hirasawa, Y. Imamura et al., "Retinal dysfunction and progressive retinal cell death in SOD1-deficient mice," American Journal of Pathology, vol. 172, no. 5, pp. 1325-1331, 2008.

[11] Y. Zhang, C. Davis, G. K. Sakellariou et al., "CuZnSOD gene deletion targeted to skeletal muscle leads to loss of contractile force but does not cause muscle atrophy in adult mice," The FASEB Journal, vol. 27, no. 9, pp. 3536-3548, 2013.

[12] T. Yoneda, T. Tomofuji, M. Kunitomo et al., "Preventive effects of drinking hydrogen-rich water on gingival oxidative stress and alveolar bone resorption in rats fed a high-fat diet," Nutrients, vol. 9, no. 1, p. 64, 2017.

[13] W. Li, B. Jiang, X. Cao, Y. Xie, and T. Huang, "Protective effect of lycopene on fluoride-induced ameloblasts apoptosis and dental fluorosis through oxidative stress-mediated caspase pathways," Chemico-Biological Interactions, vol. 261, pp. 2734, 2017.

[14] D. H. Lee, B. S. Lim, Y. K. Lee, and H. C. Yang, "Effects of hydrogen peroxide $\left(\mathrm{H}_{2} \mathrm{O}_{2}\right)$ on alkaline phosphatase activity and matrix mineralization of odontoblast and osteoblast cell lines," Cell Biology and Toxicology, vol. 22, no. 1, pp. 39-46, 2006.

[15] T.-T. Wu, L.-F. Li, R. Du, L. Jiang, and Y.-Q. Zhu, "Hydrogen peroxide induces apoptosis in human dental pulp cells via caspase-9 dependent pathway," Journal of Endodontia, vol. 39, no. 9, pp. 1151-1155, 2013.

[16] Y. Wang, O. Andrukhov, and X. Rausch-Fan, "Oxidative stress and antioxidant system in periodontitis," Frontiers in Physiology, vol. 8, p. 910, 2017.

[17] B.-J. Lee, M.-Y. Chan, H.-Y. Hsiao, C.-H. Chang, L.-P. Hsu, and P.-T. Lin, "Relationship of oxidative stress, inflammation, and the risk of metabolic syndrome in patients with oral cancer," Oxidative Medicine and Cellular Longevity, vol. 2018, 7 pages, 2018.

[18] H. Nojiri, Y. Saita, D. Morikawa et al., "Cytoplasmic superoxide causes bone fragility owing to low-turnover osteoporosis and impaired collagen cross-linking," Journal of Bone and Mineral Research, vol. 26, no. 11, pp. 2682-2694, 2011.

[19] X. D. Wang, E. A. Gillen, M. C. H. van der Meulen, and X. G. Lei, "Knockouts of Se-glutathione peroxidase-1 and $\mathrm{Cu}, \mathrm{Zn}$ superoxide dismutase exert different impacts on femoral mechanical performance of growing mice," Molecular Nutrition \& Food Research, vol. 52, no. 11, pp. 1334-1339, 2008. 
[20] G. Kharkwal, V. Chandra, I. Fatima, and A. Dwivedi, “Ormeloxifene inhibits osteoclast differentiation in parallel to downregulating RANKL-induced ROS generation and suppressing the activation of ERK and JNK in murine RAW264.7 cells," Journal of Molecular Endocrinology, vol. 48, no. 3, pp. 261270, 2012.

[21] N. Mody, F. Parhami, T. A. Sarafian, and L. L. Demer, "Oxidative stress modulates osteoblastic differentiation of vascular and bone cells," Free Radical Biology \& Medicine, vol. 31, no. 4, pp. 509-519, 2001.

[22] Y. Yamaguchi, H. Kanzaki, Y. Katsumata et al., "Dimethyl fumarate inhibits osteoclasts via attenuation of reactive oxygen species signalling by augmented antioxidation," Journal of Cellular and Molecular Medicine, vol. 22, no. 2, pp. 1138-1147, 2018.

[23] Y. Yin, X. Xue, Q. Wang, N. Chen, and D. Miao, "Bmil plays an important role in dentin and mandible homeostasis by maintaining redox balance," American Journal of Translational Research, vol. 8, no. 11, pp. 4716-4725, 2016.

[24] X. Zhou, Z. Wang, Y. Ni, Y. Yu, G. Wang, and L. Chen, "Suppression effect of $\mathrm{N}$-acetylcysteine on bone loss in ovariectomized mice," American Journal of Translational Research, vol. 12, pp. 731-742, 2020.

[25] K. Yagi, “Assay for blood plasma or serum," Methods in Enzymology, vol. 105, pp. 328-331, 1984.

[26] F. L. Muller, W. Song, Y. Liu et al., "Absence of CuZn superoxide dismutase leads to elevated oxidative stress and acceleration of age-dependent skeletal muscle atrophy," Free Radical Biology \& Medicine, vol. 40, no. 11, pp. 1993-2004, 2006.

[27] M. M. Matzuk, L. Dionne, Q. Guo, T. R. Kumar, and R. M. Lebovitz, "Ovarian function in superoxide dismutase 1 and 2 knockout mice," Endocrinology, vol. 139, no. 9, pp. 40084011, 1998.

[28] M. Almeida, E. Ambrogini, L. Han, S. C. Manolagas, and R. L. Jilka, "Increased lipid oxidation causes oxidative stress, increased peroxisome proliferator-activated receptor-gamma expression, and diminished pro-osteogenic Wnt signaling in the skeleton," The Journal of Biological Chemistry, vol. 284, pp. 27438-27448, 2009.

[29] R. A. Márquez Hernández, J. Ohtani, T. Fujita et al., "Sex hormones receptors play a crucial role in the control of femoral and mandibular growth in newborn mice," European Journal of Orthodontics, vol. 33, no. 5, pp. 564-569, 2011.

[30] J. Oiry, P. Mialocq, J. Y. Puy et al., "NAC/MEA conjugate: a new potent antioxidant which increases the GSH level in various cell lines," Bioorganic \& Medicinal Chemistry Letters, vol. 11, no. 9, pp. 1189-1191, 2001.

[31] G. Raghu, M. Berk, P. A. Campochiaro et al., "The multifaceted therapeutic role of N-Acetylcysteine (NAC) in disorders characterized by oxidative stress," Current Neuropharmacology, vol. 19, 2020.

[32] F. Balszuweit, G. Menacher, A. Schmidt et al., "Protective effects of the thiol compounds GSH and NAC against sulfur mustard toxicity in a human keratinocyte cell line," Toxicology Letters, vol. 244, pp. 35-43, 2016.

[33] Y.-C. Cheng, J.-M. Sheen, W. L. Hu, and Y.-C. Hung, "Polyphenols and oxidative stress in atherosclerosis-related ischemic heart disease and stroke," Oxidative Medicine and Cellular Longevity, vol. 2017, 16 pages, 2017.

[34] T. O. Tobore, "On the central role of mitochondria dysfunction and oxidative stress in Alzheimer's disease," Neurological Sciences, vol. 40, no. 8, pp. 1527-1540, 2019.
[35] P. L. Chiang, H. L. Chen, C. H. Lu et al., "Interaction of systemic oxidative stress and mesial temporal network degeneration in Parkinson's disease with and without cognitive impairment," Journal of Neuroinflammation, vol. 15, no. 1, p. 281, 2018.

[36] M. Olguín-Albuerne and J. Morán, "ROS produced by NOX2 control in vitro development of cerebellar granule neurons development," ASN Neuro, vol. 7, no. 2, 2015.

[37] K. Zhu, J. X. Yi, Y. J. Xiao et al., "Impaired bone homeostasis in amyotrophic lateral sclerosis mice with muscle atrophy," The Journal of Biological Chemistry, vol. 290, no. 13, pp. 80818094, 2015.

[38] C. Li, F. Wang, R. Zhang, P. Qiao, and H. Liu, "Comparison of proliferation and osteogenic differentiation potential of rat mandibular and femoral bone marrow mesenchymal stem cells in vitro," Stem Cells and Development, vol. 29, no. 11, pp. 728-736, 2020.

[39] A. Mavropoulos, R. Rizzoli, and P. Ammann, "Different responsiveness of alveolar and tibial bone to bone loss stimuli," Journal of Bone and Mineral Research, vol. 22, no. 3, pp. 403410, 2007.

[40] S. R. Vora, E. D. Camci, and T. C. Cox, "Postnatal ontogeny of the cranial base and craniofacial skeleton in male C57BL/6J mice: a reference standard for quantitative analysis," Frontiers in Physiology, vol. 6, p. 417, 2015.

[41] W. Yang and S. Hekimi, "Two modes of mitochondrial dysfunction lead independently to lifespan extension in Caenorhabditis elegans," Aging Cell, vol. 9, no. 3, pp. 433-447, 2010.

[42] R. Liang and S. Ghaffari, "Stem cells, redox signaling, and stem cell aging," Antioxidants \& Redox Signaling, vol. 20, no. 12, pp. 1902-1916, 2014.

[43] S. Dwivedi, A. Sharma, B. Patrick, R. Sharma, and Y. C. Awasthi, "Role of 4-hydroxynonenal and its metabolites in signaling," Redox Report, vol. 12, no. 1, pp. 4-10, 2013.

[44] D. A. Brown, M. A. Aon, C. R. Frasier et al., "Cardiac arrhythmias induced by glutathione oxidation can be inhibited by preventing mitochondrial depolarization," Journal of Molecular and Cellular Cardiology, vol. 48, no. 4, pp. 673679, 2010.

[45] J. Yang, Y. J. Park, H. R. Zhang et al., "Feed-forward signaling of TNF-alpha and NF-kappaB via IKK-beta pathway contributes to insulin resistance and coronary arteriolar dysfunction in type 2 diabetic mice," American Journal of Physiology. Heart and Circulatory Physiology, vol. 296, no. 6, pp. H1850-H1858, 2009.

[46] M. S. Zhou, I. H. Schulman, and L. Raij, "Vascular inflammation, insulin resistance, and endothelial dysfunction in saltsensitive hypertension: role of nuclear factor kappa B activation," Journal of Hypertension, vol. 28, no. 3, pp. 527-535, 2010.

[47] P. Patlevič, J. Vašková, P. Švorc, L. Vaško, and P. Švorc, “Reactive oxygen species and antioxidant defense in human gastrointestinal diseases," Integrative Medicine Research, vol. 5, no. 4, pp. 250-258, 2016.

[48] D. S. Tunstall Pedoe, "Sudden death risk in older athletes: increasing the denominator," British Journal of Sports Medicine, vol. 38, no. 6, pp. 671-672, 2004.

[49] N. S. Zinkevich and D. D. Gutterman, "ROS-induced ROS release in vascular biology: redox-redox signaling," American Journal of Physiology. Heart and Circulatory Physiology, vol. 301, no. 3, pp. H647-H653, 2011. 
[50] C. Yaning, T. Xin, and Y. Qinsheng, "Studies on stability of SOD," Pharmaceutical Biotechnology, vol. 6, pp. 164-167, 1999.

[51] G. Yan, Y. Guo, J. Guo, Q. Wang, C. Wang, and X. Wang, "NAcetylcysteine attenuates lipopolysaccharide-induced osteolysis by restoring bone remodeling balance via reduction of reactive oxygen species formation during osteoclastogenesis," Inflammation, vol. 43, no. 4, pp. 1279-1292, 2020.

[52] A. Vincent and M. Crozatier, "Neither too much nor too little: reactive oxygen species levels regulate Drosophila hematopoiesis," Journal of Molecular Cell Biology, vol. 2, no. 2, pp. 74-75, 2010.

[53] J. S. Johansen, A. K. Harris, D. J. Rychly, and A. Ergul, "Oxidative stress and the use of antioxidants in diabetes: linking basic science to clinical practice," Cardiovascular diabetology, vol. 4, no. 1, p. 5, 2005.

[54] M. Schieber and N. S. Chandel, "ROS function in redox signaling and oxidative stress," Current Biology, vol. 24, no. 10, pp. R453-R462, 2014. 